\title{
Spatial Quadrature Modulation for Visible Light Communication in Indoor Environment
}

\author{
Diana W. Dawoud*, Fabien Héliot*, Muhammad Ali Imran*†, Rahim Tafazolli* \\ * Institute of communication systems, Guildford GU2 7XH, UK \\ $\dagger$ School of Engineering, University of Glasgow, UK
}

\begin{abstract}
In this paper, a novel low-complexity and spectrally efficient modulation scheme for visible light communication (VLC) is proposed. Our new spatial quadrature modulation (SQM) is designed to efficiently adapt traditional complex modulation schemes to VLC; i.e. converting multi-level quadrature amplitude modulation ( $M$-QAM), to real-unipolar symbols, making it suitable for transmission over light intensity. The proposed SQM relies on the spatial domain to convey the orthogonality and polarity of the complex signals, rather than mapping bits to symbol as in existing spatial modulation (SM) schemes. The detailed symbol error analysis of SQM is derived and the derivation is validated with link level simulation results. Using simulation and derived results, we also provide a performance comparison between the proposed SQM and SM. Simulation results demonstrate that SQM could achieve a better symbol error rate (SER) and/or data rate performance compared to the state of the art in $\mathrm{SM}$; for instance a $E_{b} / N_{o}$ gain of at least $5 \mathrm{~dB}$ at a SER of $10^{-4}$.
\end{abstract}

Index Terms-Visible light communications, direct detection, Intensity modulation, spatial modulation.

\section{INTRODUCTION}

Visible light communication (VLC), which once sounded like a futuristic concept, has now morphed into an emerging technology. The potential of this technology has been recognized by academia and industry and, hence, heavy investment has recently been made to develop it further [1]. The main catalyst behind the increasing interest in VLC technology is the deployment of light emitting diodes (LEDs) as access points for wireless connectivity. These LEDs are ecologically friendly and one of today's most energy-efficient technologies, hence enabling green wireless communication. However, their low intrinsic modulation bandwidth (BW) is limiting VLC from achieving extremely high data rates. Furthermore, adopting complex modulations such as $M$-QAM and quadrature phase shift keying (QPSK) to mitigate this limited BW is not feasible, due to the signaling waveform in VLC. It should be recalled that in VLC the input data is predominantly intensity modulated and transmitted across an optical channel, then the optical power is directly detected and converted into electrical current by using a photo detector (PD). This signaling strategy imposes constrains upon the transmitted signal to be real valued and positive, leading to the need to develop efficient solutions to make the complex modulations suitable for intensity modulation/direct detection (IM/DD) systems.
To mitigate the aforementioned challenges, several multiinput multi-output (MIMO) techniques have been proposed in the literature. Schemes such as spatial modulation (SM) have been proposed to overcome the limited BW [2]. Other schemes such as optical space modulation (OSM) have been proposed to transmit bipolar signals [3]. SM exploits the spatial domain to transform information bits by modulating the index of the antenna. This technique completely avoids inter-channel interference (ICI) by only activating one antenna at each time instance. Therefore, boosting the data rate through this scheme would require drastic increase in the number of transmitting antennas (data rate increases with the logarithm of the antenna number). On the other hand, OSM employs the spatial domain as a mean to transfer the polarity of the signal. However, OSM requires converting the complex signal to a real-valued signal using the Hermitian symmetry and the inverse fast fourier transformation (IFFT). This modification reduces the spectral efficiency (SE) by a factor of two.

Accordingly, this paper aims at improving the work in [3] by considering in-phase and quadrature transmission in the spatial domain, but without the need for Hermitian symmetry or IFFT, which are detrimental to the SE and complexity, respectively. In our proposed scheme, entitled SQM, the use of the spatial domain is expanded to convey orthogonality along with polarity to transform the complex signals to real-unipolar signals without sacrificing the SE, as opposed to [3]. Though it might be misleading to use the term quadrature modulation since it refers conventionally to the combined modulation of the amplitude and the phase, here the quadrature term refers to the combined modulation of the amplitude and the spatial index, wehere the phase is mapped to the spatial index. Therfore, It should be also noted here that spatial domain is not exploited to transmit any information bits. Consequently, in contrast to [2], [4] increasing the modulation order does not increase the number of required LEDs, as it will be shown in section II. Furthermore, in certain scenarios our SQM scheme can correct transmission error, i.e. even if the index of the antenna was incorrectly detected, the transmitted symbol can still be perfectly recovered without introducing any symbol error. In addition, our proposed SQM scheme implements a low complexity receiver, unlike the joint detection in [2] where an increase of one modulation order yields a two-fold increase in complexity, that is composed of two maximum likelihood 
detection (MLD) phases. Phase one detects the index of the transmitter, while phase two detects the amplitude, which limits the increase in complexity for high modulation order.

The rest of the paper is organized as follows. In section II, the optical channel topology and the developed approach are described. Section III presents the analysis of the SER for our proposed SQM scheme. Section IV presents simulation results and comparisons. Finally, section V concludes the paper.

\section{System Model For LED-BASEd Orthogonal TRANSMISSION}

\section{A. Scenario of interest}

In this paper, we consider an indoor VLC system installed inside a room with dimension (Lenght $\times$ Width $\times$ Height) 4.0 $\times 4.0 \times 3.0 \mathrm{~m}$ as it is shown in Fig. 1. The VLC system is composed of $N_{t}$ LEDs that are installed at a height of $z=$ $2.25 \mathrm{~m}$ and oriented downwards to point straight down from the ceiling. The receiving $N_{r}$ PDs are placed on a table with a height of $z=0.75 \mathrm{~m}$. At each LED, the input electrical current is IM and transmitted over an $N_{t} \times N_{r}$ optical MIMO channel $\mathbf{H}$. The optical power at the PDs is directly converted back into electrical current, such that $\mathbf{y}=\mathbf{H x}+\mathbf{n}$, where $\mathbf{y}=\left[y_{1}, y_{2}, \cdots N_{r}\right]$ are the received electrical currents. Vector $\mathbf{x}=\left[x_{1}, x_{2}, \cdots N_{t}\right]$ represents the transmitted optical signals. The sum of the ambient shot light noise and thermal noise, $\mathbf{n}=\left[n_{1}, n_{2}, \cdots N_{r}\right]$, is modeled as a real valued additive white Gaussian noise (AWGN) with zero mean and variance $\sigma_{n}^{2}$ i.e. $\mathcal{N}\left(0, \sigma_{n}^{2}\right)$. The MIMO matrix $\mathbf{H}$ is given by

$$
\mathbf{H}=\left[\begin{array}{cccc}
h_{1,1} & h_{1,2} & \ldots & h_{1, N_{t}} \\
\vdots & \vdots & \ddots & \vdots \\
h_{N_{r}, 1} & h_{N_{r}, 2} & \ldots & h_{N_{r}, N_{t}}
\end{array}\right],
$$

where the line-of-sight (LOS) optical path loss between the $i$ th PD and the $j$-th LED, $h_{i, j}$, is estimated by assuming that the optical radiation is similar to a Lambertian radiation pattern [5]. Therefore, $h_{i, j}$ can be expressed as

$$
h_{i, j}= \begin{cases}\frac{w_{i}^{2} A_{i}}{d_{i, j}^{2} \sin ^{2}\left(\psi_{c_{i}}\right)} R_{o}\left(\phi_{i, j}\right) \cos \left(\psi_{i, j}\right) & 0 \leq \psi_{i, j} \leq \psi_{c_{i}} \\ 0 & \psi_{i, j}>\psi_{c_{i}}\end{cases}
$$

where $A_{i}$ is the detector area, $w_{i}$ denotes the refractive index, and $d_{i, j}$ is the distance between the $i$-th PD and the $j$-th LED. Moreover, $\phi_{i, j}$ and $\psi_{i, j}$ are the irradiance and incidence angles, respectively, and $\psi_{c_{i}}$ is the field of view (FOV) semiangle of the PD. Here, the channel direct current (DC) luminous intensity gain, $R_{o}(\phi)$, is given by $R_{o}\left(\phi_{i, j}\right)=$ $[(m+1) / 2 \pi] \cos ^{m}\left(\phi_{i, j}\right)$, where $m=-\ln (2) / \ln \left(\cos \phi_{1 / 2_{j}}\right)$ and denotes the order of the Lambertian emission, Here, $\phi_{1 / 2_{j}}$ represents the transmitter semi-angle. Furthermore, we assume an ideal synchronization of all the links and that time dispersion is negligible [6]. This is due to the fact that LEDs and PDs are in close proximity, as it is depicted in Fig. 1.

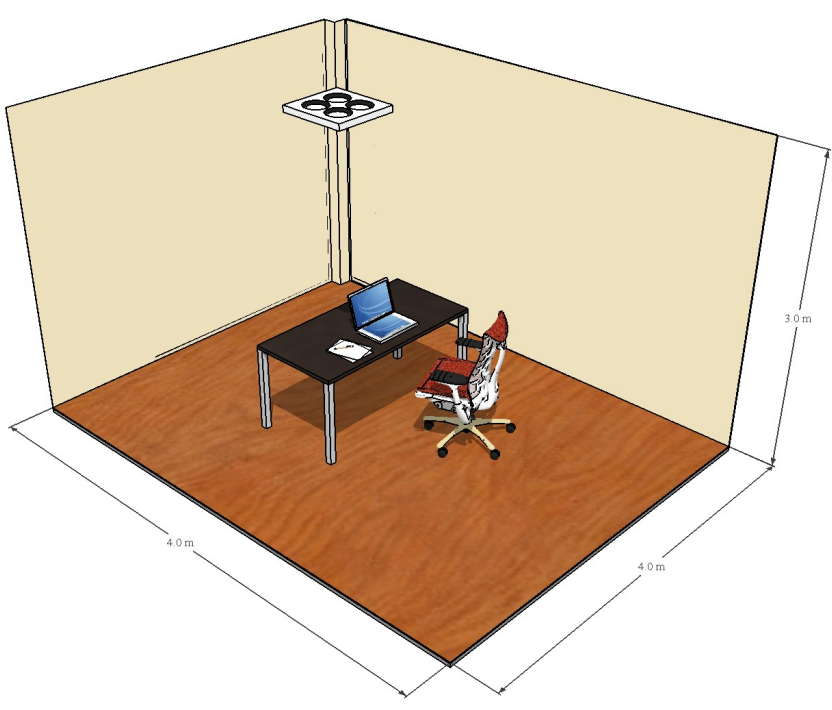

Fig. 1. Smart lighting office

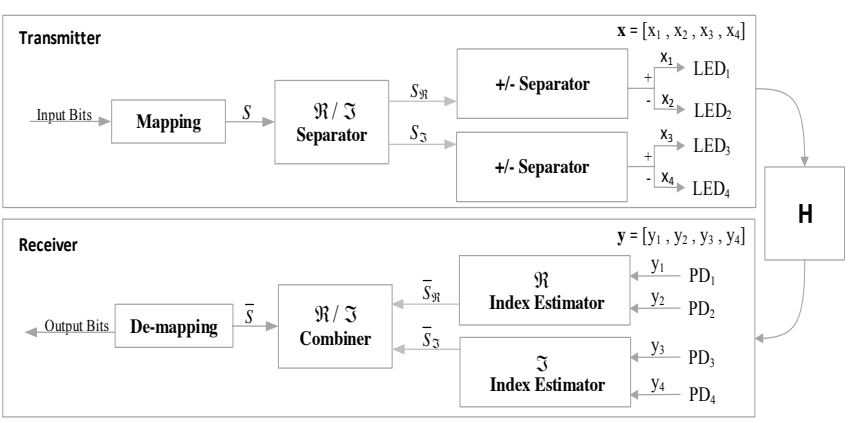

Fig. 2. Block Diagram of the SQM Scheme.

\section{B. SQM Transceiver Structure}

In this paper, we propose a novel multiple antenna transmission scheme for VLC, known as SQM. Contrary to the traditional SM scheme of [4] for which the number of LEDs increases with the modulation order, only four LEDs are required in our SQM scheme regardless of the order of the modulation scheme, as it is depicted in Fig. 2. It can be seen from Fig. 2 that after the data bits have been mapped into a symbol $S$, the latter is split into in-phase and quadratic parts; $S_{\Re}$ and $S_{\Im}$. Subsequently, the polarity of $S_{\Re}$ and $S_{\Im}$ determines the LED from which these parts of $S$ are then transmitted, as it is illustrated in Table I. The LEDs are then used to modulate the signals $\widetilde{S}_{\Re}$ and $\widetilde{S}_{\Im}$ (through IM) and transmit them across the optical wireless channel $\mathbf{H}$. Though the number of required transmitting LEDs in our scheme is $N_{t}=4$, naturally, the number of active LEDs at each symbol time, $T_{s}$, is two and this applies to the PDs as well. This is unlike the state of the art spatial division multiplexing (SDM) which requires all transmitters to be activated at each $T_{s}$ [7].

In SQM, as in [8], perfect alignment between the transmit- 
TABLE I

SIGNAL TO LED ALLOCATION

\begin{tabular}{|c|c|c|}
\hline \hline Signal Polarity & LED No. & Transmitted Signal \\
\hline \hline$S_{\Re}>0$ & 1 & $\widetilde{S}_{\Re}=S_{\Re}$ \\
\hline$S_{\Re}<0$ & 2 & $\widetilde{S}_{\Re}=\left|S_{\Re}\right|$ \\
\hline$S_{\Im}>0$ & 3 & $\widetilde{S}_{\Im}=S_{\Im}$ \\
\hline$S_{\Im}<0$ & 4 & $\widetilde{S}_{\Im}=\left|S_{\Im}\right|$ \\
\hline
\end{tabular}

ters and the receivers is required to totally avoid ICI. This perfect alignment between the transmitters and the receivers can be established in practice if the distance between transmitters satisfies the following condition, which can be easily derived by using trigonometry rules and (1), such that

$$
d_{T x} \geq \sqrt{2\left(h \tan \left(\psi_{c_{j}}\right)\right)^{2}-\left(d_{R x}\right)^{2}} .
$$

Assuming that $\psi_{c_{j}}=15^{\circ}, h=2.25-0.75=1.5 \mathrm{~m}$ and the spacing of the receivers is such that $d_{R X}=0.1 \mathrm{~m}$, which would allow the PDs array to be implemented into typical laptop computers, the distance between the transmitters, $d_{T x}$, should be more than $\geq 0.559 \mathrm{~m}$ to allow for perfect alignment between the LEDs and the PDs. Under this condition, the channel matrix will become a diagonal matrix, such that

$$
\mathbf{H}_{d_{T X}=0.6 m}=10^{-4}\left(\begin{array}{cccc}
0.7976 & 0 & 0 & 0 \\
0 & 0.7976 & 0 & 0 \\
0 & 0 & 0.7976 & 0 \\
0 & 0 & 0 & 0.7976
\end{array}\right) .
$$

Though this specific alignment is not practical for mobile receivers, it can be implemented for static receivers, e.g. video conferencing. In addition, in cases where perfect alignment is not feasible, pre-coding at the LEDs can be used to compensate for the misalignment [9].

At the receiver, MLD is employed to estimate the indices of the active LEDs and the amplitude of transmitted symbols. It should be recalled that transmitting $S_{\Re}$ would require either the first or the second LED to be active. Therefore, to detect the index of the transmitting LED of $S_{\Re}$, the following detection metric is defined

$$
\bar{b}=\arg \max _{b}\left(y_{b}\right)
$$

where $\mathrm{b} \in(1,2)$, and $\bar{b}$ is the estimated transmitting LED index of $S_{\Re}$. Consequently, the real part of the received signal will be detected based on the following rule

$$
\bar{S}_{\Re}=\left\{\begin{aligned}
y_{1} & \text { if } \bar{b}=1 ; \\
-y_{2} & \text { if } \bar{b}=2 .
\end{aligned}\right.
$$

Similarly for $S_{\Im}$, the decision rule is defined as

$$
\bar{k}=\arg \max _{k}\left(y_{k}\right)
$$

where $k \in(3,4)$, and $\bar{k}$ is the estimated transmitting LED index of $S_{\Im}$. Thus, the imaginary part of the received signal will be estimated as

$$
\bar{S}_{\Im}=\left\{\begin{aligned}
j y_{3} & \text { if } \bar{k}=3 ; \\
-j y_{4} & \text { if } \bar{k}=4 ;
\end{aligned}\right.
$$

It should be noted that at this stage, contrary to SM, no information bits are de-mapped or decoded. Afterward, the $\Re / \Im$ combiner recombines $\bar{S}_{\Re}$ and $\bar{S}_{\Im}$ for each symbol, such that $\bar{S}=\bar{S}_{\Re}+\bar{S}_{\Im}$. Finally, the detected signal $\bar{S}$ is passed through a de-mapper in order to obtain the output bits.

The most noteworthy feature of the proposed receiver is that it relies on a two phases MLD as it is previously explained. This architecture is less complex compared to the joint MLD proposed in [2]; especially for higher order modulation. For example, considering a modulation order of $M=256$, the received signal y should be compared against 256 possible transmitted symbols in joint detection. Whereas in our proposed scheme, the first detection stage is comprised of two different sets which only requires a comparison between two signals to determine the indices of the transmitting symbols. The key feature of this stage is that its complexity is independent of the modulation order. In the second stage, each MLD will compare the amplitude of the received signals against 8 possibilities only. Therefore, a $93 \%$ (from 256 to 18 comparisons) reduction in complexity is achieved through our proposed scheme as compared to joint detection when $M=256$.

\section{ANALYTICAL SER OF SQM}

In our SQM modulation scheme, a block of information bits is mapped into a constellation point in the signal domain, e.g. $M$-QAM or QPSK. Hence, the in-phase and quadraturephase components of the signal could have positive or negative polarities, as well as multiple amplitude levels. Then each of these components are mapped to a new signal space points in the spatial domain to achieve unipolar amplitudes. Therefore, the first step in the detection process is to establish from which antennas the real and imaginary parts, respectively, have been transmitted. Then the second step in the process is to detect the amplitude (absolute value) of the transmitted symbols. In order to calculate the SER performance of our SQM scheme, we have assumed that the two decoding phases are independent, however, this is not generally the case and the general SER expression for SQM, which is provided in Proposition 1, is not the exact SER, but a tight upper-bound. However, for the case of $M=4$, the derived expression is exact as will be shown in section IV. Note that to simplify the notation, we assumed in the following that $\mathbf{H}$ is unit normalized. 
Proposition 1: In the general case, the SER of our SQM modulation can be tightly upper bounded by

$$
\begin{array}{r}
S E R=\frac{1}{M} \sum_{m=1}^{M} 1-\left\{Q\left(\frac{-\widetilde{S}_{\Re_{m}}}{\sqrt{2 \sigma_{n}^{2}}}\right) P_{c}\left(\bar{S}_{\Re_{m}}\right)\right. \\
\left.Q\left(\frac{-\widetilde{S}_{\Im_{m}}}{\sqrt{2 \sigma_{n}^{2}}}\right) P_{c}\left(\bar{S}_{\Im_{m}}\right)\right\},
\end{array}
$$

where $Q(\cdot)$ is the Q-function. Furthermore, $P_{c}\left(\bar{S}_{\Re_{m}}\right)$ and $P_{c}\left(\bar{S}_{\Im_{m}}\right)$ are the probabilities to correctly estimate the amplitude of $\bar{S}_{\Re_{m}}$ and $\bar{S}_{\Im_{m}}$, respectively, when the indices of the transmitters are correctly decoded.

In the case of $M=4$, since $\bar{S}_{\Re_{m}}=\bar{S}_{\Im_{m}}=\sqrt{E_{b}}$, given that $E_{b}$ is the energy per bit, and $P_{c}\left(\bar{S}_{\Re_{m}}\right)=P_{c}\left(\bar{S}_{\Im_{m}}\right)=$ $Q(-\sqrt{2} \gamma)$, where $\gamma=\sqrt{E_{b} / 2 \sigma_{n}^{2}}$, (4) can be simplified. Furthermore, for this case it will be shown in subsection III-B that under certain conditions even if the indices of the transmitting LEDs were detected erroneously, our SQM scheme would be capable of recovering the symbol correctly. Hence, the general SER formulation in (4) can be tightened to get the exact SER for the case of $M=4$ as

$$
\begin{aligned}
S E R= & 1-Q^{2}(-\gamma) Q^{2}(-\sqrt{2} \gamma)-0.5 Q(\gamma) Q(\sqrt{2} \gamma) \\
& \times[0.5 Q(\gamma) Q(\sqrt{2} \gamma)+2 Q(-\gamma) Q(-\sqrt{2} \gamma)],
\end{aligned}
$$

Note that details regarding the derivations of (4) and (5) are provided in subsections III-A. and III-B, respectively.

\section{A. General Case}

To derive the SER, the pairwise error probability, $P_{e}\left(\bar{S}_{m}\right)$, is computed by considering all the possible signal constellation points, $\bar{S}_{m}(m=1,2,3, \ldots M)$, such that

$$
S E R=\sum_{m=1}^{M} P\left(\bar{S}_{m}\right) P_{e}\left(\bar{S}_{m}\right)
$$

where $P\left(\bar{S}_{m}\right)$ is the probability of each symbol. Note that $P_{e}\left(\bar{S}_{m}\right)=1-P_{c, c}\left(\bar{S}_{m}, T_{x_{c}}\right)$, here, $P_{c, c}\left(\bar{S}_{m}, T_{x_{c}}\right)$ stands for the probability to correctly decode $\bar{S}_{m}$ when $\bar{b}$ and $\bar{k}$ are correctly decoded $\left(T_{x_{c}}\right)$, where $T_{x_{c}}$ is the event when both $\bar{b}$ and $\bar{k}$ are correctly detected. Obviously, $P_{c, c}\left(\bar{S}_{m}, T_{x_{c}}\right)$ corresponds to the joint probability that the estimate of the amplitude of $\bar{S}_{\Re_{m}}$ and $\bar{S}_{\Im_{m}}$ are correctly decoded when $\bar{b}$ and $\bar{k}$ are correctly estimated and those probabilities will be denoted by $P_{c, c}\left(\bar{S}_{\Re_{m}}, \bar{b}_{c}\right)$ and $P_{c, c}\left(\bar{S}_{\Im_{m}}, \bar{k}_{c}\right)$, respectively. Taking into account that $\bar{S}_{\Re_{m}}$ and $\bar{S}_{\Im_{m}}$ are statically independent, then

$$
P_{c, c}\left(\bar{S}_{m}, T_{x_{c}}\right)=P_{c, c}\left(\bar{S}_{\Re_{m}}, \bar{b}_{c}\right) P_{c, c}\left(\bar{S}_{\Im_{m}}, \bar{k}_{c}\right) .
$$

The following discussion will consider the derivation of $P_{c, c}\left(\bar{S}_{\Re_{m}}, \bar{b}_{c}\right)$ and it can obviously be extended to $P_{c, c}\left(\bar{S}_{\Im_{m}}, \bar{k}_{c}\right)$. Let $P_{c}(\bar{b})$ and $P_{c}\left(\bar{S}_{\Re_{m}}\right)$ be the probabilities to correctly decoded $\bar{b}$ and the amplitude of $\bar{S}_{\Re_{m}}$, respectively. Based on the assumption that the two decoding phases are independent, then

$$
P_{c, c}\left(\bar{S}_{\Re_{m}}, \bar{b}_{c}\right)=P_{c}(\bar{b}) P_{c}\left(\bar{S}_{\Re_{m}}\right) .
$$

It should be recalled that $\bar{b}$ is estimated according to (2). This corresponds to locating the PD whose associated signal results in the greatest magnitude among a set of two signals. One of the signals is $\mathcal{N}\left(0, \sigma_{n}^{2}\right)$, since the noise is assumed to be AWGN with zero mean and variance $\sigma_{n}^{2}$. Whereas the other signal is $\mathcal{N}\left(\widetilde{S}_{\Re_{m}}, \sigma_{n}^{2}\right)$. To illustrate this point, let us assume that $S_{\Re}$ is positive. Then, the first signal in the set of the two received signals; $y_{1}$ and $y_{2}$, is a Gaussian random variable (RV) with mean $\widetilde{S}_{\Re_{m}}$ and variance $\sigma_{n}^{2}$. Thus, the probability density function (pdf) for $y_{1}$ is

$$
f_{y_{1}}(x)=\frac{1}{\sqrt{2 \pi \sigma_{n}^{2}}} e^{-\frac{\left(x-\widetilde{S}_{\Re_{m}}\right)^{2}}{2 \sigma_{n}^{2}}}
$$

The second signal $y_{2}$ is then $\mathcal{N}\left(0, \sigma_{n}^{2}\right)$ and its pdf is given by

$$
f_{y_{2}}(z)=\frac{1}{\sqrt{2 \pi \sigma_{n}^{2}}} e^{-\frac{\left(z^{2}\right)}{2 \sigma_{n}^{2}}}
$$

Consequently, the probability of correctly detecting the index of the real part $(\bar{b})$ occurs when the amplitude of the signal received at the first receiver is larger than the amplitude of the signal at the second receiver i.e. $x>z$. In other words, when $x-z>0$. It is known that the pdf of a difference of two normally distributed RVs, $x$ and $z$, with means and variances $\left(\mu_{x}, \sigma_{x}\right)$ and $\left(\mu_{z}, \sigma_{z}\right)$, respectively, is given by

$$
f_{x-z}(u)=\frac{e^{-\frac{\left[u-\left(\mu_{x}-\mu_{z}\right)\right]^{2}}{2\left(\sigma_{x}^{2}+\sigma_{z}^{2}\right)}}}{\sqrt{\left(2 \pi\left(\sigma_{x}^{2}+\sigma_{z}^{2}\right)\right)}} .
$$

Therefore, by inserting $\sigma_{x}^{2}=\sigma_{z}^{2}=\sigma_{n}^{2}, \mu_{x}=\widetilde{S}_{\Re_{m}}$ and $\mu_{z}=0$ in $(11), P_{c}(\bar{b})$ can be evaluated by

$$
\begin{aligned}
P_{c}(\bar{b}) & =\int_{0}^{\infty} \frac{e^{-\frac{\left(u-\widetilde{S}_{\Re_{m}}\right)^{2}}{4 \sigma_{n}^{2}}}}{\sqrt{4 \pi \sigma_{n}^{2}}} d u \\
& =Q\left(\frac{-\widetilde{S}_{\Re_{m}}}{\sqrt{2 \sigma_{n}^{2}}}\right) .
\end{aligned}
$$

The next step in evaluating $P_{c, c}\left(\bar{S}_{\Re_{m}}, \bar{b}_{c}\right)$ is to compute $P_{c}\left(\bar{S}_{\Re_{m}}\right)$. The latter depends on the constellation layout at the transmitter and it can be easilty calculated for traditional constellations, e.g. $M$-QAM, $M$-QPSK, based on existing literature [10]. For instance, let us consider a 4-QPSK constellation, where the distance between the points in the signal constellation diagram is $2 \sqrt{E_{b}}$, the $P_{c}\left(\bar{S}_{\Re_{m}}\right)$ can be represented as reported in [10] by

$$
P_{c}^{4}\left(\bar{S}_{\Re_{m}}\right)=Q(-\sqrt{2} \gamma) \text {. }
$$

Similarly, $P_{c, c}\left(\bar{S}_{\Im_{m}}, \bar{k}_{c}\right)$ can be given by

$$
P_{c, c}\left(\bar{S}_{\Im_{m}}, \bar{k}_{c}\right)=Q\left(\frac{-\Im_{m}}{\sqrt{2 \sigma_{n}^{2}}}\right) P_{c}\left(\bar{S}_{\Im_{m}}\right) .
$$

Next, the SER for the general case can be expressed as

$$
S E R=\frac{1}{M} \sum_{m=1}^{M} 1-P_{c, c}\left(\bar{S}_{\Re_{m}}, \bar{b}_{c}\right) P_{c, c}\left(\bar{S}_{\Im_{m}}, \bar{k}_{c}\right),
$$


when assuming equiprobable symbols. Equation (4) can be obtained by substituting (8), (12), (13) and (14) in (15).

\section{B. Case of $M=4$}

Now, we analyze the SER of our SQM scheme for the case of $M=4$ and show that in certain conditions if the indices of the transmitting LEDs were received erroneously, it is still possible to recover the original transmitted symbol. Let $P_{c, e}\left(\bar{S}_{m}, T_{x_{e}}\right)$ be the probability to correctly decode $\bar{S}_{m}$ when one or both of $\bar{b}$ and $\bar{k}$ are incorrectly decoded. To illustrate how $P_{c, e}\left(\bar{S}_{m}, T_{x_{e}}\right)$ is derived, an example is provided in the following. Let us consider that the transmitted symbol is $S_{1}=\sqrt{E_{b}}(1+j)$, then $P_{e}(\bar{b})$, the probability that the transmitting LED index of the real part is incorrectly detected can be found by using

$$
\begin{aligned}
P_{e}(\bar{b}) & =1-P_{c}(\bar{b}) \\
& =Q(\gamma) .
\end{aligned}
$$

In other words, this is the probability that the AWGN signal at the second receiver, given in (10), is larger than the received signal, given in (9), at the first receiver. That also holds in the special case when both signals, $y_{1}$ and $y_{2}$, are negative, e.g. when $y_{1}=-0.3$ and $y_{2}=-0.2$. In this case, since $y_{2}>y_{1}$ the estimated index of the transmitting LED will be incorrectly detected as $\bar{b}=2$. To summarize, $S_{\Re}$ being positive in our example, the active LED (according to Table I) is the first LED and $\bar{b}$ should be detected as 1 and not 2 . However, because of the AWGN, the index of the LED was detected incorrectly; $\bar{b}=2$. Then according to the rule in (3), $\bar{S}_{\Re}$ will be estimated as $-y_{2}$. Based on this rule and since $y_{2}<0$ the detected signal will have a positive value i.e. in our example $\bar{S}_{\Re_{1}}$ will be detected as $-y_{2}=-(-0.2)=0.2$. Thus this will cause $\bar{S}_{\Re_{1}}$ to be shifted back to the correct quadratic plane of the constellation, which therefore, will lead to detect the amplitude correctly. Hence, the probability of detecting $\bar{S}_{\Re_{1}}$ correctly when $\bar{b}$ is incorrectly decoded, $P_{c, e}\left(\bar{S}_{\Re_{1}}, \bar{b}_{e}\right)$, occurs when both the amplitude of the AWGN and desired received signals are lower than zero. Hence,

$$
\begin{aligned}
P_{c, e}\left(\bar{S}_{\Re_{1}}, \bar{b}_{e}\right) & =P_{e}(\bar{b}) P(x<0) P(z<0) \\
& =0.5[Q(\gamma) Q(\sqrt{2} \gamma)] .
\end{aligned}
$$

Following the same methodology, the probability of detecting $\bar{S}_{\Im_{1}}$ correctly when $\bar{k}$ is incorrectly decoded, $P_{c, e}\left(\bar{S}_{\Im_{1}}, \bar{k}_{e}\right)$, can be found to be equivalent to (17). Having (8), (12), (13), (14) and (17), the computation of $P_{c, e}\left(\bar{S}_{1}, T_{x_{e}}\right)$ becomes straightforward. Given the following disjoint causes:

- $\bar{b}_{e}$ and $\bar{k}_{e}$ were incorrectly detected and led to correctly recover $\bar{S}_{\Re}$ and $\bar{S}_{\Im}$ simultaneously, which corresponds to the probability $P_{c, e \Re, \Im}\left(\bar{S}_{1}\right)=$ $P_{c, e}\left(\bar{S}_{\Re_{1}}, \bar{b}_{e}\right) P_{c, e}\left(\bar{S}_{\Im_{1}}, \bar{k}_{e}\right)$

- $\bar{b}_{e}$ or $\bar{k}_{e}$ was incorrectly detected and led to correctly detect either $\bar{S}_{\Re}$ or $\bar{S}_{\Im}$ and that results in the probabilities $P_{c_{\Re}, e_{\Im}}\left(\bar{S}_{1}\right)=P_{c, c}\left(\bar{S}_{\Re_{1}}, \bar{b}_{c}\right) P_{c, e}\left(\bar{S}_{\Im_{1}}, \bar{k}_{e}\right)$ and $P_{c_{\Im}, e_{\Re}}\left(\bar{S}_{1}\right)=P_{c, e}\left(\bar{S}_{\Re_{1}}, \bar{b}_{e}\right) P_{c, c}\left(\bar{S}_{\Im_{1}}, \bar{k}_{c}\right)$.
This implies that $P_{c, e}\left(\bar{S}_{1}, T_{x_{e}}\right)$ can be expressed as by

$$
\begin{aligned}
P_{c, e}\left(\bar{S}_{1}, T_{x_{e}}\right)= & P_{c, e_{\Re, \Im}}\left(\bar{S}_{1}\right)+P_{c_{\Im}, e_{\Re}}\left(\bar{S}_{1}\right) \\
& +P_{c_{\Re}, e_{\Im}}\left(\bar{S}_{1}\right) .
\end{aligned}
$$

This is a significant finding, that highlights a major difference between SM and SQM. In the former, the estimated index of the transmitting LED will be de-mapped directly to the information bits, therefore, an error in the recovered bits will occur. However, in SQM, as shown in the above formulation, since demodulation is postponed till the symbol is completely recovered, an enhancement in the SER is achieved.

Consequently, the formulation of the SER for $M=4$ is obtained by subtracting (18) to (15) and knowing that $S_{\Re_{m}}=$ $S_{\Im_{m}}=\sqrt{E_{b}}$ for all the constellation points when $M=4$, such that

$$
\begin{aligned}
S E R_{4}= & 1-\left(P_{c, c}\left(\bar{S}_{\Re_{1}}, \bar{b}_{c}\right)\right)^{2}-P_{c, e}\left(\bar{S}_{\Re_{1}}, \bar{b}_{e}\right) \\
& {\left[P_{c, e}\left(\bar{S}_{\Re_{1}}, \bar{b}_{e}\right)+2 P_{c, c}\left(\bar{S}_{\Re_{1}}, \bar{b}_{c}\right)\right] }
\end{aligned}
$$

assuming that the symbols are equiprobable. Equation (5) is finally obtained by, substituting (8), (12), (13) and (17) in (19).

\section{RESULTS AND COMPARISONS}

This section presents the SER performance of the proposed SQM transmission scheme. Starting with a comparison between the analytical SER derived in section III and the simulated SER for different modulation orders; $M=$ 4, 8, 16, 32, 64, 128 and 256. As it is shown in Fig. 3, the derived general SER in proposition 1 is a very tight upper bound of the simulated SER, especially at high bit energy-to-noise ratio $\left(\mathrm{E}_{b} / \mathrm{N}_{o}\right)$; this validates the accuracy of our analytical SER upper bound for SQM. Furthermore, It is ascertained that the theoretical and simulation results for the case of $M=4$ confirm an exact match for low and high $\mathrm{E}_{b} / \mathrm{N}_{o}$.

Next, we compare our proposed SQM scheme with SM [4]. For a fair comparison, the mean electrical power, $P_{\text {ele }}$, is maintained equal for both schemes. First the bit error rate (BER) performance for the two schemes is compared when both systems achieve the same SE, as shown in Table II (first and second lines). Here, multilevel pulse amplitude modulation ( $M$-PAM) is considered for the SM system because it is more BW efficient compared to other pulse modulation techniques such as on-off Keying and multilevel pulse position modulation. Fig. 4 shows the BER of SQM and SM. As it can be seen from Fig. 4, our proposed SQM performs better in terms of BER than SM when both systems have the same data-rate; for instance when the $\mathrm{SE}$ is set to $2 \mathrm{bits} / \mathrm{s} / \mathrm{Hz}$, our $4-\mathrm{SQM}$ scheme achieves significantly better BER performance than the existing $2 \times 2$-PAM SM scheme, i.e. by at least $7 \mathrm{~dB}$ at $10^{-4}$; furthermore, when the SE is doubled to $4 \mathrm{bits} / \mathrm{s} / \mathrm{Hz}$, our 16-SQM scheme still outperforms $4 \times 4$-PAM SM scheme by $5 \mathrm{~dB}$ at a BER of $10^{-4}$. Instead of achieving a better BER (for a given SE), our SQM scheme can be used to improve the SE (for a given BER). To demonstrate the effectiveness of SQM in providing higher SE while maintaining the same BER performance as SM, we compare our 32 and 128-SQM against 
TABLE II

SIMULATION CONFIGURATIONS

\begin{tabular}{|c|c||c|c|c|}
\hline \hline \multicolumn{2}{|c||}{ SQM } & \multicolumn{3}{c|}{ SM } \\
\hline bits/s & $M$ & bits/s & $N_{t}$ & $M$ - PAM \\
\hline \hline 2 & 4 & 2 & 2 & 2 \\
\hline 4 & 16 & 4 & 4 & 4 \\
\hline 5 & 32 & 3 & 4 & 2 \\
\hline 7 & 128 & 4 & 4 & 4 \\
\hline
\end{tabular}

the $4 \times 2$ - $M$-PAM and $4 \times 4 M$-PAM SM schemes (third and fourth lines of Table II), where all the schemes have the same number of transmitting antennas, $N_{t}=4$, but with different SE. As depicted in Fig. 4, our 32-SQM scheme provides a $50 \%$ improvement gain in SE (3 instead of $2 \mathrm{bits} / \mathrm{s} / \mathrm{Hz}$ ), but for similar BER perfomance as the $4 \times 2$-PAM SM scheme. In addition, remarkably, SQM with a constellation size of 128 could perform similarly as $4 \times 4$-SM in terms of BER, but with a $75 \%$ improvement gain in SE.

\section{CONCLUSION}

In this work, a novel transmission scheme was presented, SQM, which is utilized to extend the transmission of real and positive signaling in VLC to in-phase and quadrature dimensions. The paper also entails a detailed SER performance analysis of our SQM scheme. The significant enhancement of our proposed SQM scheme is the utilization of a fixed number of transmitting LEDs, i.e. $N_{t}=4$, regardless of the modulation order. In contrast to the existing multiple antennas scheme in literature (e.g. SM), which data rate enhancement capability increases logarithmically with the number of transmit antennas. The most noteworthy findings of the Monte Carlo simulations were that our SQM scheme requires $5 \mathrm{~dB}$ less power for the same error performance and data rate as compared to SM. In addition, it was found that SQM could convey higher unit BW data rates; more than $2 \mathrm{bit} / \mathrm{s} / \mathrm{Hz}$, under the same BER performance when compared to its SM counterpart. Furthermore, results demonstrated that the derived analytical SER matches closely with simulation results. In future work, the effect of ICI on the BER performance would be investigated; in addition, techniques such as pre-coding algorithms would be introduced to mitigate the effect of ICI.

\section{REFERENCES}

[1] Gow.epsrc.ac.uk, "Ultra-parallel visible light communications (up-vlc)," 2015. [Online]. Available: http://gow.epsrc.ac.uk/NGBOViewGrant. aspx?GrantRef=EP/K00042X/1

[2] S. P. Alaka, T. L. Narasimhan, and A. Chockalingam, "Generalized spatial modulation in indoor wireless visible light communication," in IEEE Global Commun. Conf., Dec. 2015, pp. 1-7.

[3] Y. Li, D. Tsonev, and H. Haas, "Non-DC-biased OFDM with optical spatial modulation," Proc. IEEE Int. Symp. Pers. Indoor Mobile Radio Commun. PIMRC, pp. 486-490, 2013.

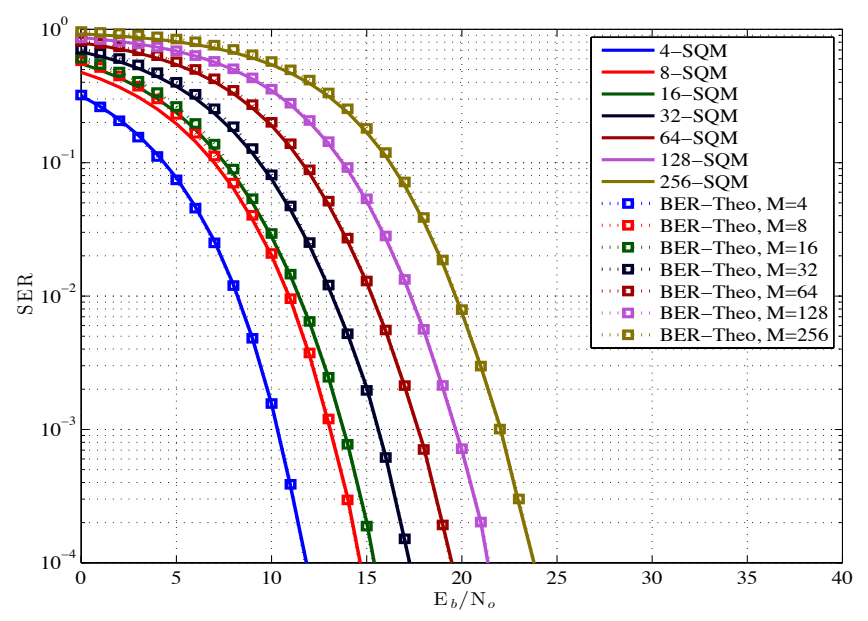

Fig. 3. Performance comparison of SQM from the Monte Carlo simulations with the theoretical SER, provided in Proposition 1, for different constellations.

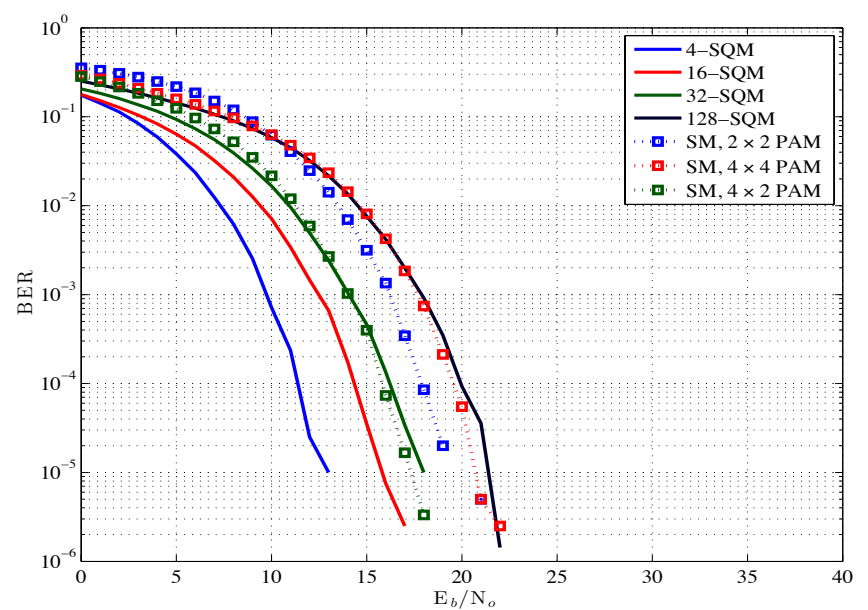

Fig. 4. Performance comparison of the proposed SQM from the Monte Carlo simulations with SM for different constellations.

[4] R. Y. Mesleh, H. Haas, S. Sinanovic, C. W. Ahn, and S. Yun, "Spatial Modulation," IEEE Trans. on Veh. Technol., vol. 57, no. 4, pp. 2228 2241, 2008.

[5] L. Zeng, D. O'Brien, H. Le-Minh, K. Lee, D. Jung, and Y. Oh, “Improvement of date rate by using equalization in an indoor visible light communication system," 4th IEEE Int. Conf. Circuits Syst. Commun. ICCSC, no. 0, pp. 678-682, 2008.

[6] T. Fath and H. Haas, "Performance comparison of mimo techniques for optical wireless communications in indoor environments," IEEE Trans. Commun., vol. 61, no. 2, pp. 733-742, Feb. 2013.

[7] G. Li, N. Bai, N. Zhao, and C. Xia, "Space-division multiplexing: the next frontier in optical communication," Adv. Opt. Photon., vol. 6, no. 4, pp. 413-487, Dec 2014. [Online]. Available: http://aop.osa.org/abstract.cfm?URI=aop-6-4-413

[8] W. O. Popoola and H. Haas, "Demonstration of the merit and limitation of generalised space shift keying for indoor visible light communications," J. Lightw. Technol., vol. 32, no. 10, pp. 1960-1965, 2014.

[9] J. Chen, Y. Hong, Z. Wang, and C. Yu, "Precoded visible light communications," in 9th Int. Conf. on Inform., Commun. and Signal Process., ICICS, 2013, Dec 2013, pp. 1-4.

[10] F. M. Andreas, Wireless Communications, 2nd ed. Wiley, 2011. 